\title{
La corteza cerebral modula la transmisión cutánea a través de los núcleos de los cordones posteriores
}

\author{
J. Mariño ${ }^{\mathrm{a}}$, J. Aguilar ${ }^{\mathrm{b}}$, C. Soto ${ }^{\mathrm{b}}$, A. Canedo ${ }^{\mathrm{b}}$ \\ THE CEREBRAL CORTEX MODULATES THE CUTANEOUS TRANSMISSION \\ THROUGH THE DORSAL COLUMN NUCLEI
}

\begin{abstract}
Summary. Introduction. The mechanisms used by the cerebral cortex to modulate the cutaneous information at prethalamic level have been scarcely studied. This article reviews experimental evidence leading to a better understanding of this issue at the level of the cuneate nucleus (Burdach nucleus). Development. The primary afferents and the corticocuneate fibers make synaptic contact with cuneothalamic neurons and with inhibitory interneurons in the middle cuneate nucleus. By stimulating the skin at different places while recording the cuneothalamic intracellular activity in anaesthetized animals with the cortex intact, with the cortex pharmacologically inactivated, or in absence of a cerebral cortex it was possible to ascertain the functional role of the corticocuneate fibers. The primary afferents activated by stimulating a particular zone of the skin induce monosynaptic excitation on a group of cuneothalamic cells at the same time at which inhibit, through intranuclear interneurons, neighboring cuneothalamic cells with unmatched receptive fields. Similarly, the corticocuneate cells receiving information from the stimulated skin further increase the excitation of the cuneothalamic neurons with matched receptive fields while inhibiting others with unmatched fields. The cortex exaggerates an excited center surrounded by an inhibited periphery thus increasing the tactile discrimination both spatially and temporally which is essential for exploratory and manipulative purposes. [REV NEUROL 2001; 33: 448-54]

Key words. Cortical modulation. Cuneate nucleus. Cuneothalamic neurons. Cutaneous transmission. Sensorimotor cortex. Ventroposterolateral thalamic nucleus.
\end{abstract}

\section{INTRODUCCIÓN}

Lapercepción del movimiento y de la posición del cuerpo depende de la actividad de las fibras aferentes procedentes de los husos neuromusculares y de los órganos tendinosos de Golgi. Las fibras aferentes que inervan los mecanorreceptores de la dermis y de la epidermis son las responsables de la percepción táctil, deldolory de la temperatura.

Los miembros anteriores de los cuadrúpedos adquieren un nuevo significado en los bípedos debido aque pierden su función locomotriz y de apoyo, en beneficio de un incremento en la capacidad exploratoria para la manipulación de los objetos del entorno. La mayor parte de la información táctil se obtiene a través de la mano, que puede considerarse comoun ‘órgano' perceptualy manipulativo. El tacto exploratorio requiere control eferente motor einformación cinestésica aferente (movimientoy posición), y produce estimulación cutánea. Sin embargo, la mayoría de los datos neurofisiológicos disponibles relacionados con la transmisión, modulación eintegración táctil se han obtenidoen animales anestesiados obien en sujetos pasivos, principalmente primates. Elloes así porque en estas condiciones se tiene un mayor control sobre los parámetros de los estímulos utilizados para elestudio del sistema.

En la piel de la mano existen cuatro tipos diferentes de mecanorreceptores táctiles, caracterizados por su posición, el tamaño de sus campos receptores y su mayoro menor gradode adaptación en respuesta a identaciones mantenidas de la piel. Los mecanorreceptores de tipo 1 se encuentran en la zona más superficial, en la

Recibido: 23.05.01. Aceptadotras revisión externa sinmodificaciones:02.06.01.

${ }^{a}$ Departamento de Medicina. Universidad de La Coruña. ${ }^{b}$ Departamentode Fisiología. Facultad de Medicina. Universidad de Santiago de Compostela. Santiago de Compostela, España.

Correspondencia: Dr. Antonio Canedo. Departamentode Fisiología. Facultad de Medicina. E-15705 Santiago de Compostela. Fax: +34981574145. E-mail: fsancala@usc.es

Agradecimientos. El trabajo experimental fue subvencionado por la Xunta de Galicia, por la DGICYT y por la CICYT.

(C) 2001, REVISTA DENEUROLOGÍA unión entre la epidermis y la dermis; poseen campos receptores pequeños y se adaptan lenta-discos de Merkel en la piel glabra y conpelo:SA1-orápidamente-corpúsculosdeMeissnerenlapiel glabra: RA1. Los mecanorreceptores de tipo 2 se encuentran más profundos, en la dermis, poseen campos receptores grandes y se adaptan con rapidez-corpúsculos de Pacini en la piel glabray con pelo: RA2-olentamente-terminaciones de Ruffini en la piel con pelo:SA2. Las terminaciones libres sobre los folículos pilosos se encuentran también en la dermis y se adaptan con rapidez.

Las fibras mielínicas primarias que inervan los mecanorreceptores son del grupo $\mathrm{A} \alpha$ y $\mathrm{A} \beta$, entran en la médula espinal y suben por los cordones posteriores (columnas dorsales) homolaterales; terminan en el núcleo de Goll o grácil(NG) las procedentes de la parte caudal del troncoy de los miembros inferiores, y en el núcleo de Burdach ocuneatus(NC), más lateral, las procedentes de los miembros superiores y parte alta del tronco. A estas poblaciones neuronales, que se extienden desde la médula oblongada hasta el bulbo, se les denomina núcleos de los cordones posteriores o núcleos de las columnas dorsales (NCD). Los NCD se relacionan, clásicamente, con la transmisión de la sensación táctil consciente (sensibilidadepicrítica), pero datos morfológicos y electrofisiológicos recientes indican que también intervienen en otros procesos, en particularen laintegración sensitivomotorabajolainfluencia de la corteza cerebral (CC) [1].

Estarevisión es unarecopilación de los datos experimentales relacionados con el procesamiento de la información táctilen los NCD. Se centrarála atención en el papel que desempeñala CCen el procesamiento de la información cutánea ascendente del NC.

\section{ORGANIZACIÓN GENERAL DE LOS NÚCLEOS DE LAS COLUMNAS DORSALES}

La mayoría de las aferencias somatosensitivas primarias ( 90\%) dirigidas a los NCD terminan en estos núcleos de forma directa. El 10\% restante lo hacen comoaferencias secundarias procedentes de neuronas espinales cercanas al canal central, que se han relacionado con procesos nociceptivos de origen visceral [2]. 
Ambas aferencias liberan glutamato y contactanmonosinápticamente, tantoconneuronas locales ointerneuronas comoconneuronas de proyección azonas extranucleares.

$\mathrm{Si}$ atendemos a criterios morfológicos y funcionales, los NCD se dividen en tres zonas, quetomancomoreferenciaelObex:

1. Unazonarostral reticulada,formadapor neuronas con grandes campos receptores musculares y articulares, y que recibe abundantes aferenciasdescendentes, desdelacortezamotoraprimariay desde elárea3a(cortezapropioceptiva).Lasneuronas de esta zona envían proyecciones contralateralmente al tálamo, tectum y pretectumy, homolateralmente, alcerebelo, olivainferiory médulaespinal[3].

2. Unazona medial, que se extiende desde niveles cercanos al Obex hasta unos 4-5 mm caudalmente. La forman una parte dorsal, con predominio de células dispuestasenagregados, que proyectanal núcleoventroposterolateral(VPL) del tálamo contralateral, y una parte ventral, formada por interneuronasinhibitorias. También existen interneuronas dispersas entre los agregados. Las células de proyección recibeninformación de receptores cutáneos localizados en la parte distal del miembrohomolateral superior (NC) oinferior(NG).Suscampos receptores son pequeñosynohay unanimidad en constatar si reciben información submodalidad-específica ono [4].Las interneuronas reciben información cutánea y propioceptiva, y sus campos receptores son mayores y más proximalesquelosdelasneuronas de proyección $[5,6]$. Asimismo, las interneuronas reciben aferencias de la formación reticular bulbary del núcleo rojo [3]. Las fibras corticonucleares también se segregan en esta parte de los NCD; mientras que las interneuronas ventrales son monosinápticamente contactadas por fibras procedentes de la corteza motora primaria y del área3a, las células de proyección, más dorsales, reciben contactos sinápticos directos de fibras procedentes mayoritariamente delárea 3b (corteza cutánea) [1,6](Fig.1).

3. Una zona reticulada caudal, cuyas neuronas se caracterizan por recibir de forma preferente información de receptores sensibles a estimulaciones cutáneas de alta frecuencia (corpúsculos de Pacini), y que envían fibras eferentes a las mismas estructuras que las neuronas de la zona rostral. Esta región es la continuación rostral de la parte medial de la médula espinal y contiene un gran número de fibras procedentes de la corteza somatosensitiva primaria(CSSP); estas últimas también se dirigen a las láminas IV-VI de los segmentos de la médula cervical alta [6,7], para modular la transmisión somatosensitivaascendente generada porlos movimientos delamano[1].

Las células de la parte medial del NC son las que han recibido una mayoratención experimental,y aellas dirigiremosnuestrointerés. Las neuronas que forman agregados [7,8] poseen campos receptores cutáneos en los dedos y establecen un 'centrocutáneo’ rodeado porotras neuronas con campos receptores cutáneos, musculares y articulares. Este centro cutáneo proyecta al VPLcontralateral, dondetambiénexiste unaregióncentraldecélulas talamocorticalescon camposreceptorescutáneos, rodeadaporcélulastalamocorticales concampos receptores de origen musculary articular. A su vez, este centro cutáneo talámico envía información a la región cutánea cortical (área 3b) en la CSSP. Estos centros cutáneos en elcuneatus, VPL y CSSP reciben una entrada dominante de las fibras de los cordones posteriores, que inervan las partes distales de las extremidades superiores. Tantoel númerode fibrasqueinervan estas regiones distales como el número de células en los sucesivos centros cutáneos a lolargode la vía, seincrementan en las especies animales con mayor capacidad manipulativa, comoen el mapache y los primates [1]. Asimismo, el incremento en la capacidad de obtener datos por las fibras cutáneas tiene un paralelismo cortical, ya que tambiénaumentaelnúmerodefibras corticofugales quemodulan lainformación ascendente en elámbitotalámicoy pretalámico[1].

Estáclaroque para estudiarel procesamiento y la modulación de la información cutánea en el núcleocuneatusmedial(NCM)se han de tener en cuenta, de forma preferente, tanto las aferencias primarias comolas influencias corticonucleares sobre las células cuneotalámicas. Estasinfluencias pueden producirdiferentesefectos, que dependen de la actividad generada intrínsecamente por las propias células del NC.

\section{PROPIEDADES INTRÍNSECAS Y RITMOGÉNESIS DE LAS CÉLULAS DEL NCM}

Datos experimentales obtenidos recientemente indican que las células del NCM poseen propiedades intrínsecas [5,9,10] similares a las descritas en el tálamo [11], lo que potencialmente las faculta para producir actividad oscilatoria sin necesidad de activación aferente.

Se conoce bien que las células t alámicas y corticales poseen dos modos de actividad: uno tónico, durante la vigilia, y otro oscilatorio, durante el sueño y bajo anestesia. En este último caso, diferentes anestésicos tienden a generar, prioritariamente, ciertos 


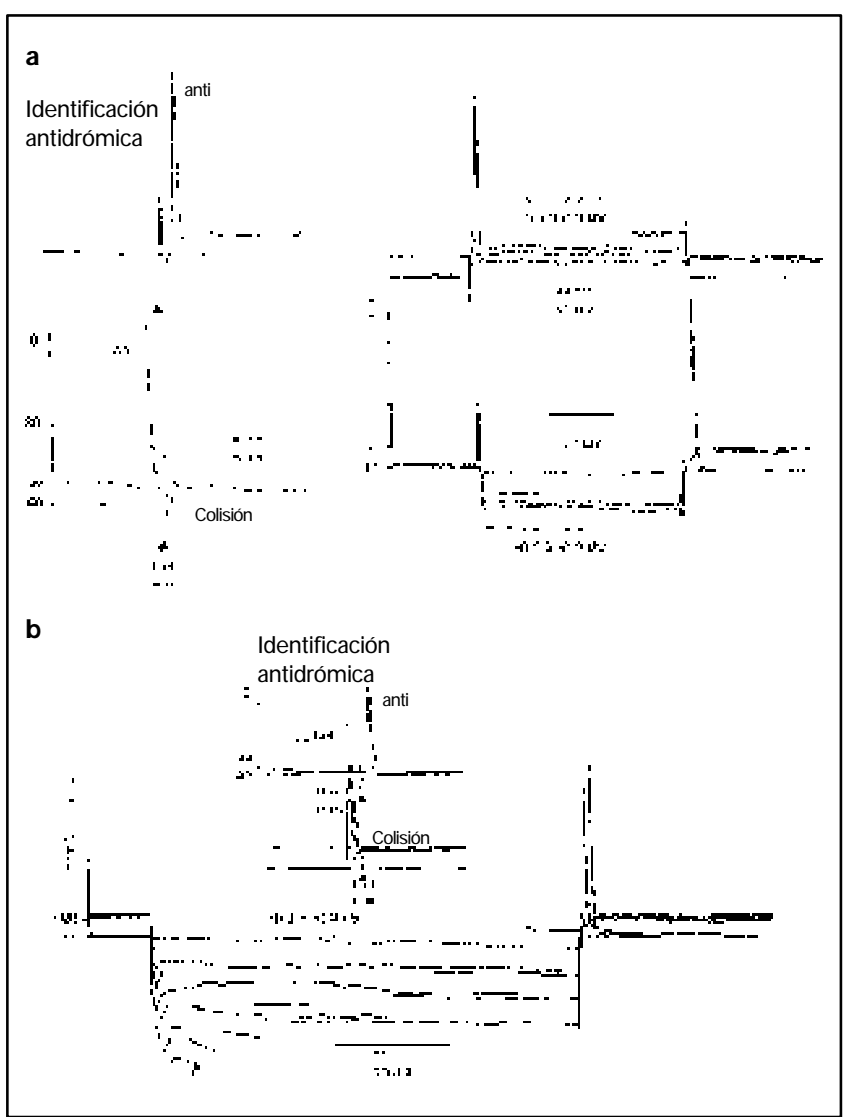

Figura 2. Propiedades intrínsecas de las neuronas cuneotalámicas. La inyección de corriente negativa a través del electrodo de registro permite diferenciar dos tipos de células: unas, cuya respuesta genera una despolarización indicativa de la presencia de corrientes lh (b, señalada por una flecha), y otras, que responden de manera pasiva (a). Sin embargo, ambos tipos de células responden al cese de la inyección de corriente y producen potenciales excitativos que pueden llevar a la aparición de espigas, lo que sugiere la existencia de canales de calcio de bajo umbral. La mayoría de las células cuneotalámicas de la parte medial del núcleo se acomodan con rapidezala inyección de corriente despolarizante, y generan nomalmente uno o unos pocos potenciales de acción(A). Las células se identifican antidrómicamente por estimulación eléctrica del lemnisco medial (LM) contralateral. La colisión-extinción que se observa entre los potenciales de acción espontáneos (esp) y los inducidos por la estimulación (anti) confirman la antidromicidad. Los artefactos de los estímulos se señalan con asteriscos.

ritmos-nembutal: ondas en huso con frecuencias intrafusales de 7-14Hz; ketamina-xylacina: ritmos deltade 1-4Hz; alfa-cloralosa: actividad punta-ondaa diferentes frecuencias-.Elmodotónicoes el que se utiliza para el procesamiento y la transmisión de lainformaciónsensorialascendente; elmodooscilatoriobloquealatransmisión sensorial hacia la corteza, yaquese generaintrínsecamente enlascélulastalámicas,y, porello, notransmitelas propiedades de los estímulos periféricos [11]. De acuerdo con estaidea, las neuronas pretalámicas nointervendrían nien la generación nien el mantenimiento de los ritmos tálamo-corticotalámicos, característicos de los estados de sueño y que también se observan bajo anestesia. Sinembargo, muchas delascélulas delos NCDpresentanactividad espontánearítmica, tanto en el animal anestesiado [1,4,5,12,13], como en preparadosin vitro [9], como en preparaciones de ratas recién nacidas con la médula espinal y el tallocerebral aislados [10].

Aligualquelascélulas talamocorticales, las neuronas cuneotalámicas del NCM poseen una corriente catiónica mixta(Ih) que se activa con la hiperpolarización [5,9,10], una corriente de calcio de bajo umbral $\left(\mathrm{I}_{\mathrm{T}}\right)$ [5] y unacorrientedepotasio $\left(\mathrm{I}_{\mathrm{A}}\right)$ que se activacon

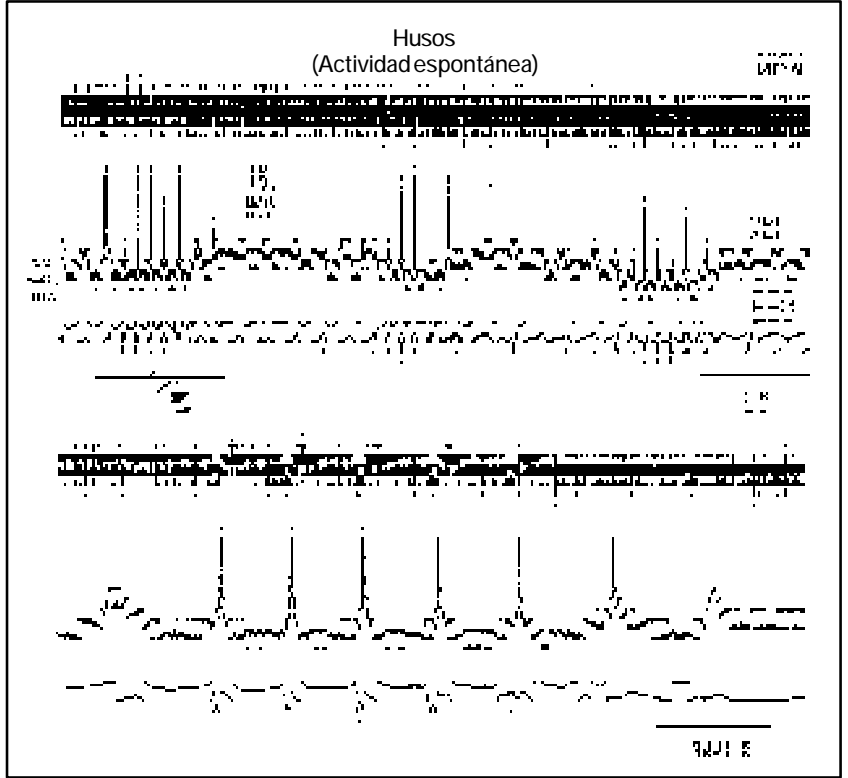

Figura 3. La actividad ńtmica generada en los circuitos tálamo-corticotalámicos se transmite al núcleo cuneatus. Los ritmos en huso, registrados con un macroelectrodo en laCSM (EEG)e intracelularmenteen el núcleo ventroposterolateral (VPL) del tálamo, se reflejan en la actividad de masa, registrada en el núcleo cuneatus medial (NCM) contralateral. La porción del registro subrayada se expande en la parte inferior de la figura (señalada por una flecha).

despolarizaciones, que se preceden dehiperpolarizaciones [9]. Estas corrientes facultan a las neuronas delcuneatus para generaractividadintrínsecaen ausenciadeestimulaciónaferente, talcomoseha observadoen sus fibras de proyecciónallemniscomedial(LM) [14] y en las propias neuronas delNC [15]. Enlafigura 2 se muestranlas respuestas de dos células cuneotalámicasalainyeccióndecorriente a través delelectrodo de registro. Una de ellas genera una despolarización a la inyección de corriente hiperpolarizante-marcada por unaflechaenlafigura 2b-,indicativadelapresenciadecorrientes $\mathrm{I}_{\mathrm{h}}$. Lasdoscélulasrespondencondespolarizacioneslentasquepueden llevaralageneración deespigas al cese delainyección decorriente negativa, loque sugiere que estas excitaciones seproducen porla activación de corrientes de calcio de bajo umbral $\left(\mathrm{I}_{\mathrm{T}}\right)[5]$.

Los ritmos en huso $(7-14 \mathrm{~Hz})$ se generan en el núcleo reticular talámico, los ritmos delta (1-4Hz) los producen las células talamocorticales y los ritmos lentos $(<1 \mathrm{~Hz})$ se originan en la CC [11]. La cortezaimpone estos ritmos en aquellas zonas subcorticales conlas que contacta monosinápticamente, que incluyenelNC $[1,16]$ yel tálamo[16]. En la figura 3 se muestra un ejemplo de la transmisión de las ondas en huso, desde la CC al NCM. Dada la similitud en la disposición neuronal del VPL y del NMC-las células de proyección rodeadas y mezcladas con interneuronas inhibitorias-, se espera que los ritmos intrínsecos que se observan en el tálamo puedan también producirse a nivel del NMC. Si ello es así, entonces las neuronas del NCM deben presentar actividad rítmica espontáneasincontribución de laCC. Datos recientes obtenidosen el gato anestesiado en nuestro laboratorio indican que ocurre de esta manera [12]. La figura 4 muestra un ejemplo de actividad rítmica dentro del intervalo delta obtenida en fibras lemniscales deun gato sin corteza frontoparietal.Lafibrarepresentadapresentabauncamporeceptorcutáneoenlapartemás distaldelmiembro contralateral anterior; por tanto, era una fibra procedente de una célula de proyección del NC. Durante el sueño y la anestesia, sin embargo, laCCimpone sus ritmos de actividadenlazona subcor- 


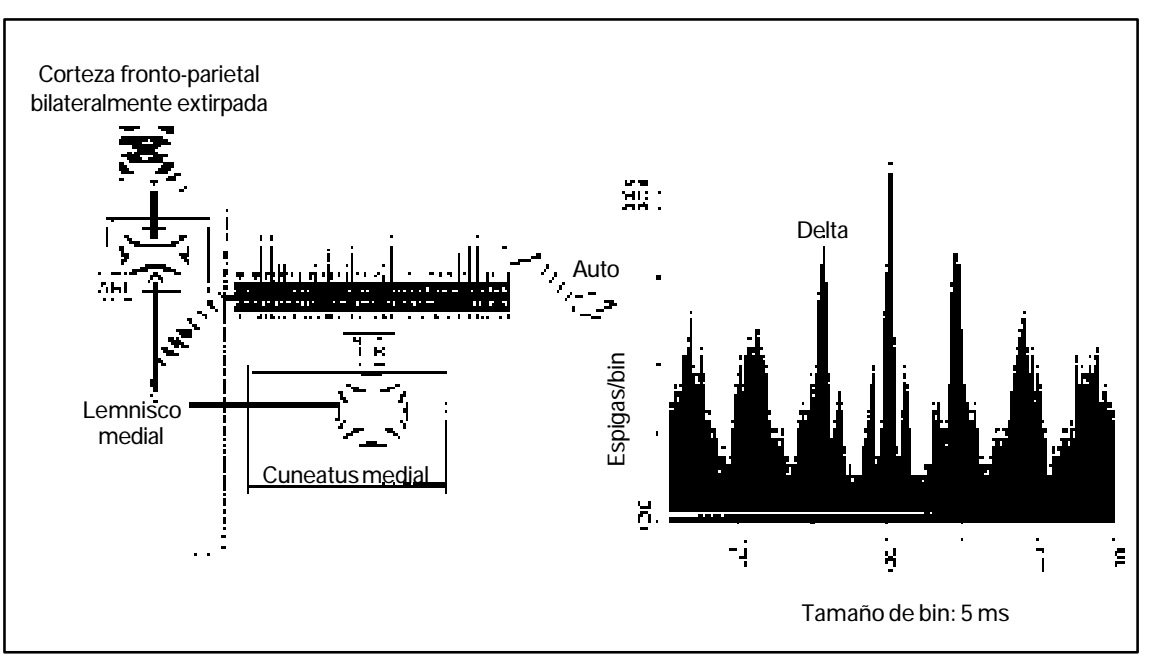

Figura 4. La actividad rítmica cuneotalámica sobrevive a la extirpación cortical. El registro de la actividad extracelular unitaria de fibras del lemnisco medial con campos receptores cutáneos, en la parte más distal del miembro superior contralateral, demuestra que poseen actividad rítmica de tipo delta (1-4 Hz; histograma de autocorrelación a la derecha), después de la ablación bilateral de la corteza frontoparietal.

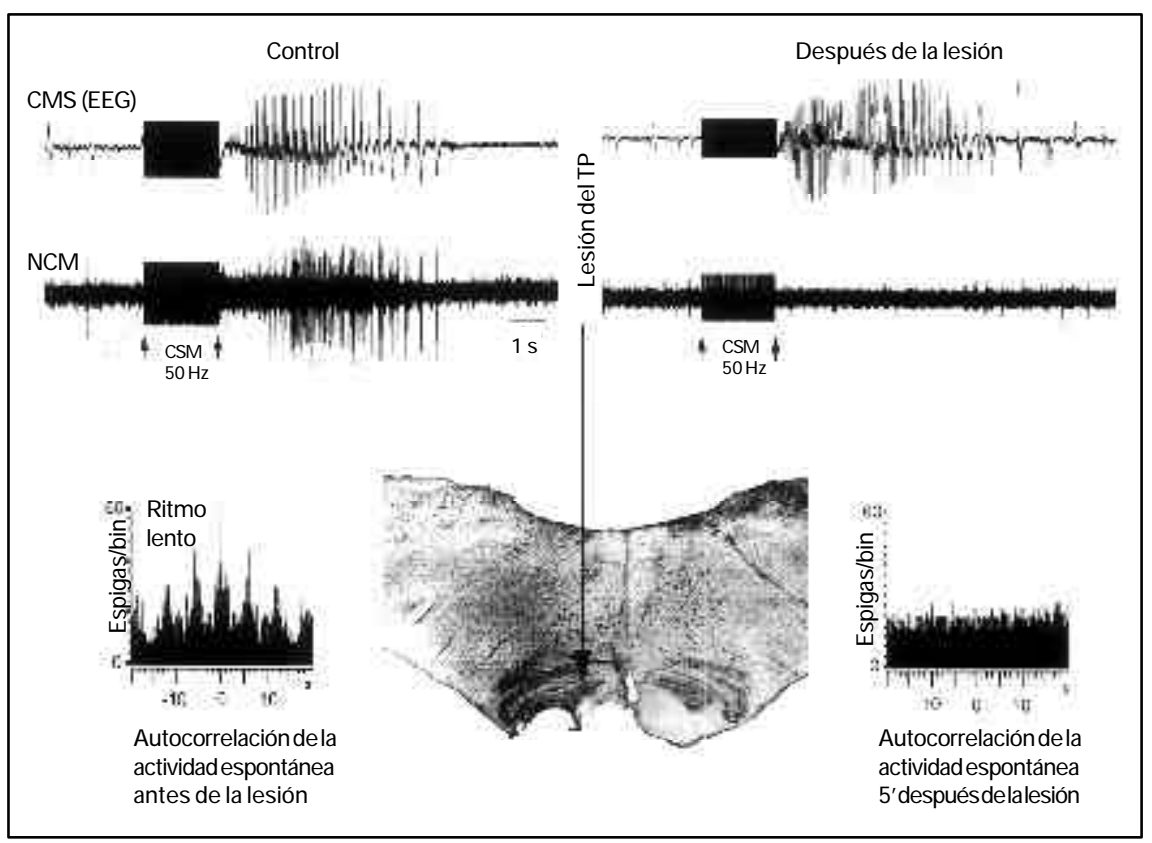

Figura 5. Las influencias corticales sobre el núcleocuneatus se ejercen a través del tracto piramidal. La estimulación eléctrica de la CSM, a $50 \mathrm{~Hz}$ durante $2 \mathrm{~s}$, produce actividad electroencefalográfica (EEG) de tipo paroxístico, que se transmite al núcleo cuneatus medial (NCM, actividad extracelular unitaria) (Control). Después de lesionar electrolíticamente el tracto piramidal (TP) en la decusación -corte frontal a nivel de la oliva inferior, con una flecha que señala la lesión-, la actividad paroxística inducida en la CC no se transmite al NCM -par de registros superiores, a la derecha. Asimismo, los ritmos lentos $(<1 \mathrm{~Hz})$ que la corteza impone sobre el NCM, en ausencia de estimulación eléctrica -histograma a la izquierda de la lesión-, desaparecen después de la lesión del TP -histograma a la derecha de la lesión-. Las flechas hacia arriba y hacia abajo en la parte superior indican el inicio y la terminación del tren de estimulación cortical.

tical, de tal forma que se filtra la actividad ascendente y prevalece la oscilatoria.

Enresumen, aunquelasneuronascuneotalámicasposeenactividadrítmicapropia,encondicionesnormalessuactividadlamodula laCC,detalformaqueduranteelsueñotantolaactividadpretalámica como la talámica entran en resonancia con la cortical. Durante la vigilia, la corteza produce efectos más discretos y favorecelaactividadascendente deinterés previamente seleccionadaporellamisma.

\section{EFECTOS PRODUCIDOS POR LA CC SOBRE \\ LAS NEURONAS \\ CUNEOTALÁMICAS DEL NCM}

Dadoque lacorteza sensoriomotora(CSM) (áreas 1, 2, 3 y 4) se proyecta de forma topográfica sobre elNC $[6,7,17]$, la sincronizaciónartificialdelasneuronas deCSSP en la zona de representación de la mano maximiza la influencia cortical sobre las células cuneotalámicas del NCM. La estimulación eléctrica cortical durante 1-2 s, a $50-100 \mathrm{~Hz}$,generaactividadparoxísticaque se transmite al NCM a través del tracto piramidal, ya que los efectosinducidos por lacorteza desaparecen después de la lesión electrolítica de este tractoen sudecusación [12]. En la figura 5 se presentan registros electrocorticográficos de la CSM y registros multiunitarios en el NCM, obtenidos antes (izquierda) y después (derecha) de la lesión electrolítica del tracto piramidal contralateral al NCM registrado. Se observa que la actividad paroxística cortical producida por la estimulación eléctrica de la parte lateral de la corteza motora genera actividadrítmicasincronizadaenelNCM,con un retraso que varió de unos a otros animales.Lalesión de las fibras corticonucleares en la decusación del tracto piramidal eliminó la influencia cortical. En condiciones basales, la CSM impone su ritmo sobre el NCM-histograma de autocorrelación a la izquierda de la lesión, en la figura 5-, que desaparece después de la lesión del tracto piramidal-histogramade autocorrelación a la derecha de la lesión en la figura 5 .

Aunque lainfluenciacortical es mayoritariamenteexcitatoriasobrelasinterneuronaseinhibitoriasobrelascélulascuneotalámicas, también se han observado los efectos opuestos $[1,4,12,18]$. El hecho de que laCCinhibainterneuronas inhibitorias es labase fisiológica para postular fenómenos de desinhibición cortical sobre la transmisión cuneotalámica $[1,12,13]$.Esto,junto con la demostración de que la corteza también puede activar monosinápticamente neuronas cuneotalámicas $[1,4,6,12,18]$, sirve de apoyo a laidea de que la CC puede potenciar la transmisión de ciertas señales ascendentes en detrimento de otras.

La vía corticonuclearconsta de al menos dos componentes: 1 . Células cuyos axonesconducen lentamentey noalcanzan la médula espinal, y 2. Colaterales de células corticoespinales rápidas, cuyos axones terminan en la médula cervical [19]. Dado que las células corticonucleares rápidas tiendenagenerarpotenciales deacciónde forma fásica y las lentas de forma tónica, lainfluencia de la corteza sobre los NCD es dual. Las fibras corticonucleares tónicas contactanmonosinápticamente coninterneuronasinhibitorias, y producen 
unainhibiciónmantenidaeinespecíficasobrelascélulas de proyección. Sólolas aferencias primarias capaces de superarestainhibición transmitirán información al tálamo. Las fibras corticonucleares fásicas se activan por la estimulación de campos receptores específicos y potencian latransmisión delasneuronas delos NCD concamposreceptoressolapados, medianteexcitaciónmonosinápticaydesinhibición; almismotiempo, activaninterneuronasinhibitoriasintranuclearesoenlaformaciónreticularvecina[20],queinhiben neuronas cuneotalámicas concamposreceptoresnosolapados. Esto sugierequelasinterneuronas afectadas porambas poblaciones de fibras corticofugales deben serdistintas, hechoque sehade esclarecer; perose sabequelasinterneuronas delNC sonheterogéneas: unas liberan GABA, otras glicinay otras a mbos neurotransmisores [21].

Un porcentaje significativo ( 28\%) de las fibras corticonucleares (no corticoespinales) envían ramas colaterales al núcleo rojoy al núcleo reticular gigantocelular origen de fibras rubroespinales y reticuloespinales, respectivamente [19]; estodemuestra que las mismas células de la CSM no sólo se interesan por la modulación de la actividad somestésica ascendente, sino también por el control postural-a través d el sistemareticuloespinal-y del movimiento de los miembros -a través del sistema rubroespinal. Además, las fibras corticoespinales que colateralizan a los NCD terminan en la médula cervical, lo que significa que su interés se dirigealamodulación de laactividad sensoriomotorarelacionada conlosmiembrossuperiores. Sedesconocesiestasfibras corticocervicalesterminan en las láminas posteriores, eninterneuronas dela lámina VII, que, a su vez, afectan la actividad motoneuronal, en las propiasmotoneuronasoen los treslugares. Posiblementese distribuyanalastreszonasy afectentantoalaactividadmotrizdeexploración comoalaactividadsensitivageneradaporelp ropiomovimiento; esto último en la zona medulary de los NCD, ya que la actividad motrizincrementa lacapacidad de discriminación cutánea, tanto espacialcomotemporalmente.Porejemplo,sisepretendenotarel calcetín en eldedogordodel pie, la mayoría de la gentelo moverá paraincrementarladiscriminación.Lainformación periféricaseleccionadavoluntariamentealcanzalaCCysepercibeconscientemente.Paraque elloocurrasenecesitapotenciarlainformaciónde interés, al tiempo que se inhiben otras que pueden interferir. Los NCDdesempeñanunpapeldecisivoenestapotenciacióneinhibición, y ambos fenómenos los incrementa laCC.

LaCCpotencialas señales excitativas procedentes delcentro y las inhibitorias procedentes de la periferia de los campos receptores cutáneos

La estimulación mecánica $[5,22,23]$ y eléctrica $[4,5]$ de los campos receptores cutáneos produce ráfagas de potenciales de acción en las células cuneotalámicas. Estas ráfagas de 3-8 potenciales, separados por poco más de $1 \mathrm{~ms}$-pueden alcanzar frecuencias de hasta 800-900/s-, se generan por diferentes mecanismos. Éstosincluyen: 1.Potenciales postsinápticos producidos por activaciónaferente, porcolateralesrecurrentesdeaxoneslemniscales que reentran en el núcleoy por señales lemniscales que afectan a las neuronas nucleares a través de la CC [18], y 2. Las propiedades intrínsecas de membrana $[5,9,10]$, ya que las neuronas cuneotalámicas dependen del potencial de membrana paragenerar diferentestipos de actividad [5].

Resultados obtenidos en el gato anestesiado demuestran que la estimulación cutánea produce una respuesta doble en los NCD. La primera se debe a la activación producida por las aferencias ascendentes, y la segunda se genera reflejamente por la CC a través del tracto piramidal [24].La respuesta cortical apareceinespecífica en experimentos de estimulación masiva, pero puede ser

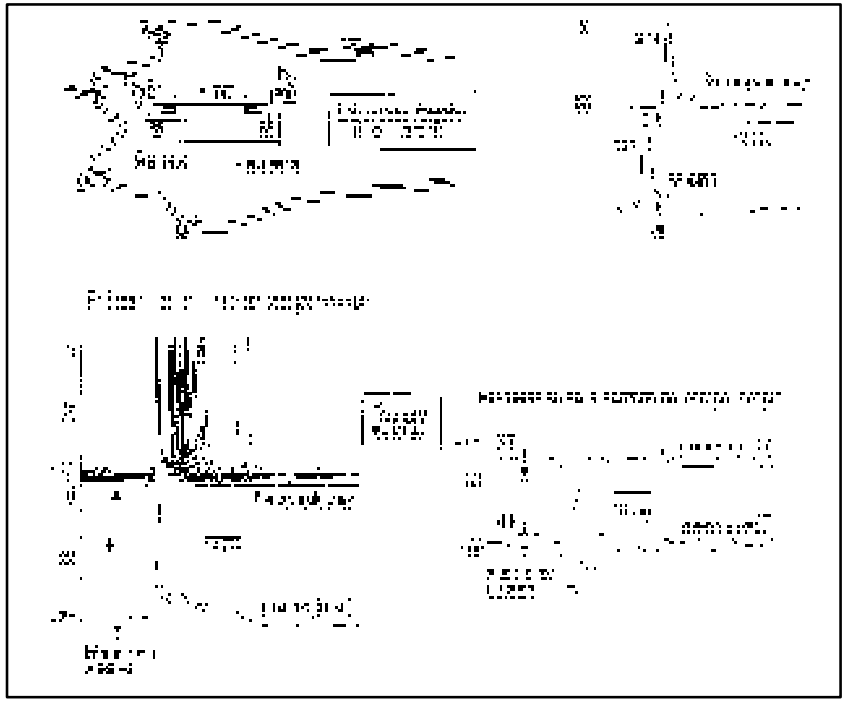

Figura 6. La estimulación en el centro y en la periferia del campo receptor produce efectos excitativos e inhibitorios, respectivamente. Una célula cuneotalámica -respuesta antidrómica y colisión con una espiga espontánea en el par de registros superiores, a la derecha del esquema- genera respuestas excitativas a la estimulación eléctrica en una zona de la piel -par de registros inferiores, a la izquierda- e inhibitorias a la estimulación en otra zona separada $15 \mathrm{~mm}$ de laanterior-parde registros inferiores, ala derecha, según se esquematiza en el dibujo de la parte superior izquierda. Los artefactos de la estimulación en el lemnisco medial (LM) se señalan con asteriscos. Tindica la intensidad de estimulación supraumbral en el centro del campo y $2 T$ y $3 T$, el doble y el triple de dicha intensidad aplicada en la periferia del campo. Se nota la similitud en las latencias de respuesta a la estimulación en el centro y en la periferia del campo.

altamente específica si se activan campos receptores discretos. Si tal como se ha supuesto, la CC incrementa la transmisión de la información relevante y elimina lairrelevante, el fenómeno debe ponerse demanifiestocuandoseactivaelcentroy/olaperiferiade loscampos receptorescutáneos de diferentes células concampos receptores solapados y sin solapar. En particular, hay que demostrar que las células del NCM activadas por lae stimulación de sus campos receptores excitadores activan célulastalamocorticales del VPL con campos receptores solapados; estos, a su vez, transmiten esta información a una población de células corticales con campos receptores también solapados.Lahipótesis predicequelas células corticales activadas por laestimulación de un camporeceptorconcreto potencian la transmisión procedente de ese campo, tanto a nivel pretalámicocomotalámico. Siasumimos lo anterior, laestimulación de las zonas vecinas a los campos receptores excitadores delasmismascélulas, nosolamentedebe producirinhibiciónlateral mediada porinterneuronas delNCMy delVPL-en este casotambién a través de las células gabérgicas del núcleo reticular talámico-, sinoque también las células corticonucleares, con campos receptores localizados en estas mismas zonas vecinas, deben potenciar esta inhibición. La corteza exageraría así, en el ámbito pretalámicoy talámico, un centro de excitación rodeadoporuna periferia de inhibición, para incrementar la discriminación de los lugares de la piel estimulados con respecto a los no estimulados.

Paraponera pruebaestahipótesis se diseñaron experimentosen gatos anestesiados, en los que se estimularon tanto el centro como la vecindad de los campos receptores excitadores de células cuneotalámicas, identificadas antidrómicamente por estimulación eléctrica del lemnisco medial contralateral.Para determinar la contribución cortical, los experimentos se realizaron en tres condiciones diferentes: 1.Conlacortezaintacta; 2.ConlaCSMinactivadacon 


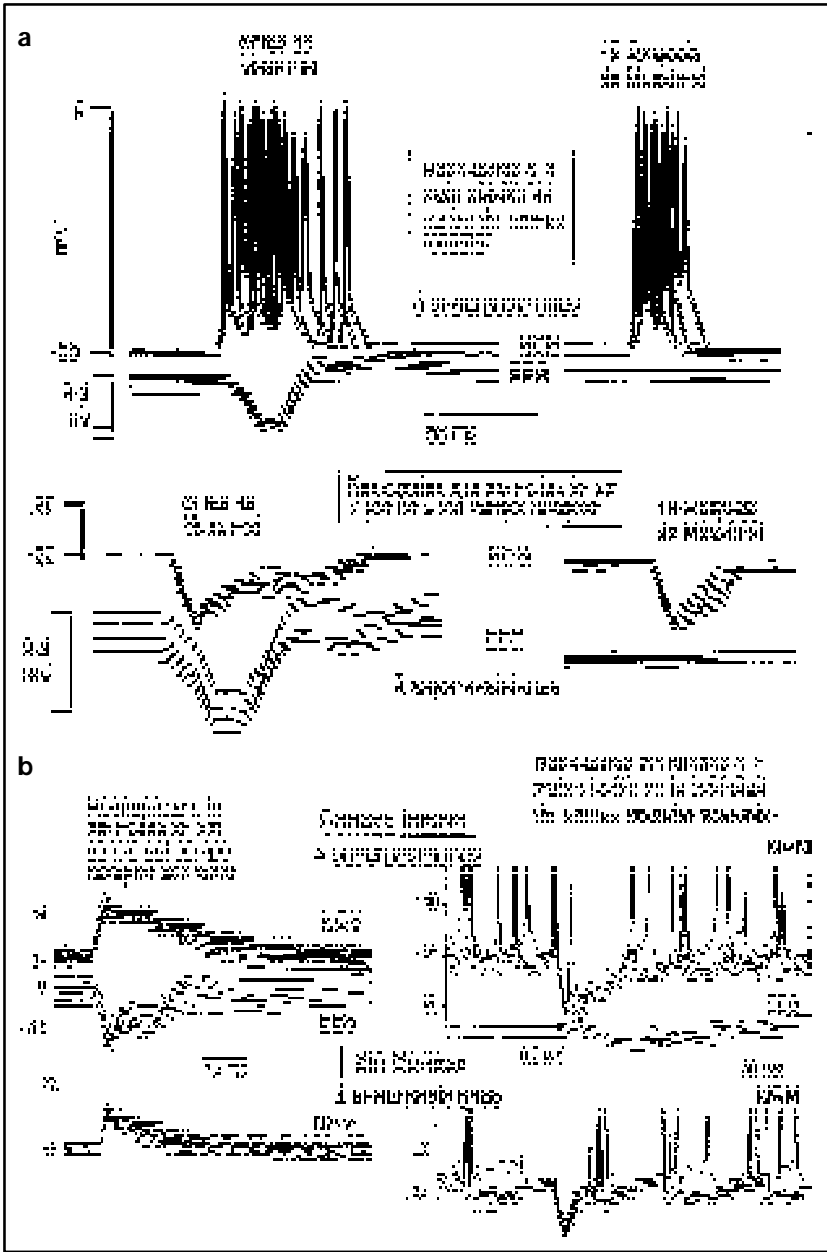

Figura 7. La CSM incrementa tanto la activación que produce la estimulación del centro del campo como la inhibición que causa la estimulación de la periferia. a) Respuestas de una misma célula cuneotalámica a la estimulación cutánea, antes -columna de la izquierda-y después -columna de la derecha- de la inactivación cortical con muscimol. La inactivación cortical redujo sensiblemente la duración de los potenciales sinápticos excitativos e inhibitorios -columna de la derecha-; b)R espuestas de cuatro células cuneotalámicas distintas a la estimulación cutánea con la CC intacta (fila superior) y después de la extirpación bilateral de la corteza frontoparietal (fila inferior). La ablación cortical redujo de forma considerable la duración de las respuestas generadas por la estimulación del centro y de la periferia del campo. La dramática reducción en amplitud de los registros EEG, después de la aplicación tópica de muscimol en los animales con CC, indican la efectividad en la inactivación cortical.

muscimol-unagonistagabérgico-,y 3.Despuésdelaextirpación bilateraldelacortezafrontoparietal [4]. Los resultados obtenidos corroboran lahipótesis planteada. Así, se observaquelaestimulacióndelcentrodelcamporeceptorcutáneoproducelaactivaciónde las células cuneotalámicas, quereciben aferencias de dichocampo, mientras que la activación de campos vecinos produce inhibición (Fig. 6). Se destaca que los efectos inhibitorios obtenidos por la estimulación de la periferia del campo presentan latencias similares, e inclusoinferiores, a los efectos excitativos generados por la estimulación delcentrodelcampo(Fig.6).Estehechosugiereque si las fibras aferentes primarias son excitativas [25] y se necesita una interneurona adicional para producir lainhibición, las fibras que contactan sinápticamente con estainterneurona son más veloces que las que lo hacen con las células de proyección.

Porotraparte, tantolainactivación comolaextirpación cor-

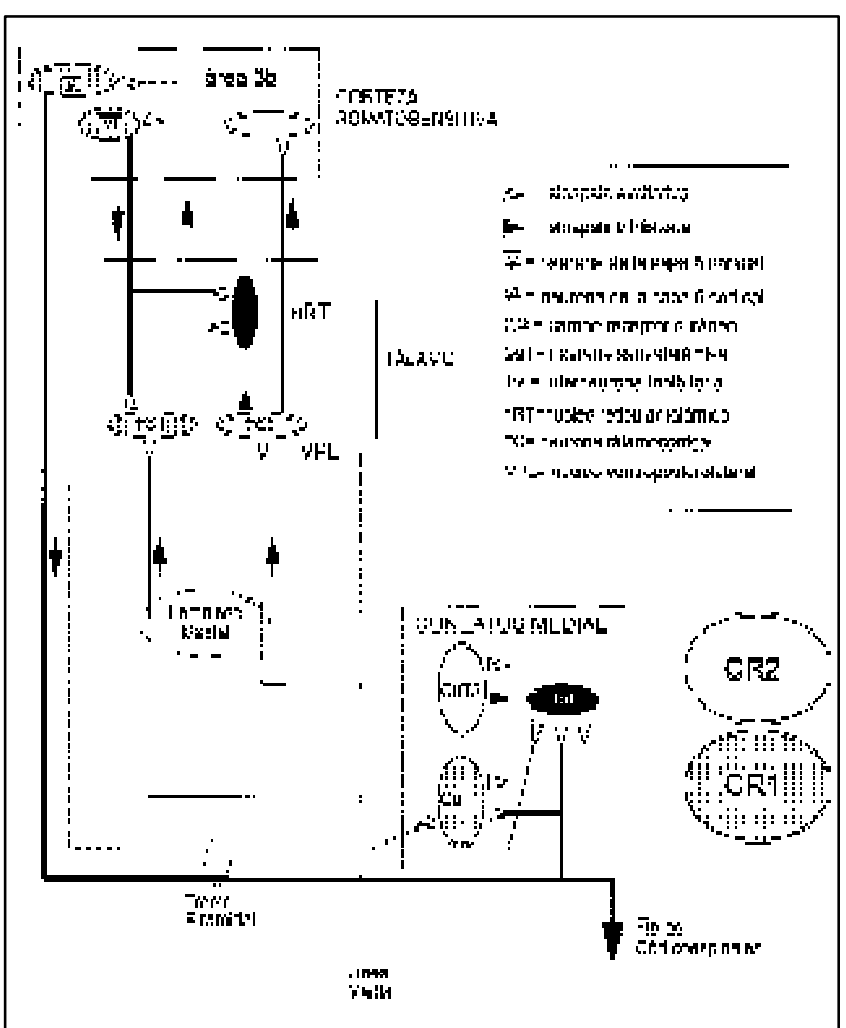

Figura 8. Interacciones centrales entre diferentes canales de transmisión cutánea. La activación de una zona discreta de la piel (p. ej., el campo receptor 1: CR1) excitalas correspondientes células cuneotalámicas (CUT1) y talamocorticales (TC1) e inhibe, a través de interneuronas inhibitorias, otras células de proyección (CuT2, TC2) que reciben información de otras zonas de la piel (p. ej., CR2). Las células corticales que se activan por la estimulación de CRI potencian la excitación ascendente procedente de CR1, tanto del cuneatus como del VPL, si bien a través de fibras corticofugales distintas -las corticotalámicas se originan en la capa VI, mientras que las de mayor recorrido proceden de la capa V. Las mismas fibras corticofugales potencian también la inhibición lateral, producida por las fibras procedentes de CR1, sobre las neuronas CUT2 y TC2.

tical redujeron la duración de los efectos excitativos e inhibitorios producidos por la estimulación del centro y de la periferia del campo, respectivamente(Fig. 7). Ello demuestra que la CC potencia la actividad de las células cuneotalámicas cuyos campos receptores se han activado y también potencia la inhibición de las neuronas cuneotalámicas vecinas cuyos campos receptores están menos activos, lo que lleva a un incremento en la discriminación espacial. Resultados similares se han obtenido en el VPLtalámico [4], lo que explica que se pueda seleccionar voluntariamente la información procedente de un lugar de la piel, y que la actividad motora incrementa dicha discriminación.

\section{CONCLUSIONES}

Deacuerdoconloexpuesto, los sectorescutáneosdelsistemasomestésico se conectan en serie a lo largo de la vía hasta la CSSP. La transmisión de la información cutánea procedente de los distintos camposreceptores setransmiteporcanalesparalelos, coninteracciones laterales entre ellos. Laexistenciade interneuronas inhibitorias tanto en elNCMcomoen el VPL-se incluyen aquílas neuronas del núcleoreticulartalámico-hace posiblelos procesos de inhibición lateral.Lasfibrasaferentesactivadasporlaestimulacióndesucampo receptor excitan neuronas cuneotalámicas, que, a su vez, excitan 
neuronastalamocorticales;porestarazón,poseencamposreceptores solapados. Al mismotiempo, las fibras aferentes activadas inhiben, a través de interneuronas, otras células vecinas de proyección con diferentes campos receptores.Las fibras corticotalámicas y corticonucleares procedentes de neuronas activadas por laestimulación del mismocampo receptor potencian tantola excitación de las células activadascomolainhibicióndelasinhibidasaambosnivelessubcor- ticales. La CC mantiene así una zona de excitación rodeada por una zona de inhibición, e incrementa tanto la discriminación espacial comolatemporal.Enesteúltimocasoporqueaumentaeltiempoque las células deproyección permanecen despolarizadas(Fig.7)y, por ello, también aumenta la capacidad celular para responder a altas frecuencias de estimulación. De esta manera, laCC es capaz de seleccionar sus propias entradas (Fig. 8).

\section{BIBLIOGRAFÍA}

1. Mariño J, Martínez L, Canedo A. Sensorimotor integration at the dorsal column nuclei. News Physiol Sci 1999; 14: 231-7.

2. Willis WD, Al-Chaer ED, Quast MJ, Westlund KN. A visceral pain pathway in the dorsal column of the spinal cord. Proc Natl Acad Sci U S A 1999; 96: 7675-9.

3. Berkley KJ, Budell RJ, Blomqvist A, Bull M. Output systems of the dorsal column nuclei in the cat. Brain Res Rev 1986; 11: 199-225.

4. Canedo A, Aguilar J. Spatial and cortical influences exerted on cuneothalamic and thalamocortical neurons of the cat. Eur J Neurosci 2000; 12: 2515-33

5. Canedo A, Martínez L, Mariño J. Tonic and bursting activity in the cuneate nucleus of the chloralose- anesthetized cat. Neuroscience 1998; 84: 603-17.

6. Cheema S, Whitsel BL, Rustioni A. The corticocuneate pathway in the cat: Relations among terminal distribution patterns, cytoarchitecture, and single neuron functional properties. Somatosensory Res 1983; 2: 169-205.

7. Kuypers HGJM, Tuerk JD. The distribution of the cortical fibers within the nuclei cuneatus and gracilis in the cat. J Anat (Lond) 1964; 98: 143-62.

8. Ramón y Cajal S. Histologie du Système Nerveux de l'Home et des Vertébrés. Vol. 1. Madrid: Consejo de Investigaciones Científicas; 1952.

9. Núñez A, Buño W. In vitro electrophysiological properties of rat dorsal column nuclei neurons. Eur J Neurosci 1999; 11: 1865-76.

10. Deuchars SA, Trippenbach T, Spyer KM. Dorsal column nuclei neurons recorded in a brain stem-spinal cord preparation: characteristics and their responses to dorsal root stimulation. J Neurophysiol 2000; 84: 1361-8.

11. Steriade M, Jones EG, McCormick DA. Thalamus. Vol. 1. Oxford: Elsevier; 1997.

12. Mariño J, Canedo A, Aguilar J. Sensorimotor cortical influences on cuneate nucleus rhythmic activity in the anesthetized cat. Neuroscience 2000; 95: 657-73.

13. Canedo A. Primary motor cortex influences on the descending and ascending systems. Progr Neurobiol 1997; 51: 287-335.

\section{LA CORTEZA CEREBRALMODULA LA TRANSMISIÓN CUTÁNEA A TRAVÉS DE LOS NÚCLEOS DE LOS CORDONES POSTERIORES}

Resumen. Introducción. Los mecanismos que utiliza la corteza cerebral para modular la información táctil a nivel pretalámico se han estudiado muy poco. Desarrollo. En este artículo recopilamos evidencias experimentales que permiten avanzar en el conocimiento de este problema a nivel del núcleo cuneatus (núcleo de Burdach). Las fibras aferentes primarias y las corticofugales contactan sinápticamente con células cuneotalámicas y con interneuronas inhibitorias en la parte medial del núcleo cuneatus. La estimulación de diferentes lugares de la piel mientras se registra intracelularmente la actividad de las neuronas cuneotalámicas en animales anestesiados y con la corteza cerebral intacta, o con la corteza inactivada farmacológicamente, o en ausencia de corteza, ha permitido el estudio del papel funcional de las fibras corticales dirigidas al núcleo cuneatus. Las fibras aferentes primarias activadas por la estimulación de una zona de la piel excitan las neuronas cuneotalámicas con las que contactan monosinápticamente e inhiben, a través de interneuronas intranucleares, otras células cuneotalámicas vecinas que reciben información de otros lugares de la piel. De la misma manera, las células corticocuneatus activadas por la estimulación de la piel incrementan la excitación de las células cuneotalámicas con campos receptores solapados e inhiben la actividad de las demás. La corteza exagera de esta forma un centro excitado rodeado por una región inhibida, lo que incrementa la capacidad de discriminación táctil tanto espacial como temporal durante la exploración y la manipulación. [REV NEUROL 2001; 33: 448-54]

Palabras clave. Corteza sensitivomotora. Modulación cortical. Neuronas cuneotalámicas. Núcleo cuneatus. Núcleo talámico ventroposterolateral. Transmisión cutánea.
14. Brown AG, Gordon G, Kay RH. A Study of single axons in the cat's medial lemniscus. J Physiol (Lond) 1974: 236: 225-46.

15. Galindo A, Krnjevic K, Schwartz S. Patterns of firing in cuneate neurons and some effects of Flaxedil. Exp Brain Res 1968; 5: 87-101.

16. Mariño J, Martínez L, Canedo A. Coupled slow and delta oscillations between cuneothalamic and thalamocortical neurons in the chloralose anesthetized cat. Neurosci Lett 1996; 219: 107-10.

17. Martínez-Lorenzana G, Machín R, Avendaño C. Definite segregation of cortical neurons projecting to the dorsal column nuclei in the rat. Neuroreport 2001; 12: 413-6.

18. Canedo A, Mariño J, Aguilar J. Lemniscal recurrent and transcortical influences on cuneate neurons. Neuroscience 2000; 97: 317-34.

19. Martínez L, Lamas JA, Canedo A. Pyramidal tract and corticospinal neurons with branching axons to the dorsal column nuclei of the cat. Neuroscience 1995; 68: 195-206.

20. Sotgiu ML, Margnelli M. Electrophysiological identification of pontomedullary reticular neurons directly projecting into dorsal column nuclei. Brain Res 1976; 103: 443-53.

21. Popratiloff A, Valtschanoff JG, Rustioni AS, Weinberg RJ. Colocalization of GABA and Glycine in the rat dorsal column nuclei. Brain Res 1996; 706: 308-12.

22. Pubols BH Jr, Haring JH, Rowinski MJ. Patterns of resting discharge in neurons of the raccoon main cuneate nucleus. J Neurophysiol 1989; 61: 1131-41.

23. Calvin WH, Loeser JD. Doublet and burst firing patterns within the dorsal column nuclei of cat and man. Exp Neurol 1975; 48: 406-26.

24. Towe AL, Zimmerman ID. Peripherally evoked cortical reflex in the cuneate nucleus. Nature 1962; 194: 1250-1.

25. Rustioni A, Weinberg RJ. The Somatosensory system. In Björklund A Hökfelt T, Swanson LW, eds. Handbook of Chemical Neuroanatomy. Vol. 7. Integrated systems of the CNS, part II. Amsterdam: Elsevier; 1989. p. 219-320.

\section{O CÓRTEX CEREBRAL MODULA A TRANSMISSÃO CUTÂNEA ATRAVÉS DOS NÚCLEOS DOS CORDÕES POSTERIORES}

Resumo. Introdução. Osmecanismos que ocórtexcerebral utilizapara modular a informação táctil a nível pré-talâmico têm sido muito pouco estudados. Desenvolvimento. Neste artigo recompilamos evidências experimentais que permitem avançarno conhecimento deste problema a nível do núcleo cuneatus (núcleo de Burdach). As fibras aferentes primárias e as córtico-eferentes contactam sinapticamente com células cuneotalâmicas e com interneurónios inibidores na parte mediana do núcleo cuneatus. A estimulação de diferentes locais cutâneos enquanto se regista intracelularmente a actividade dos neurónios nucleotalâmicos em animais anestesiados e com córtex cerebral intacto, ou com o córtex farmacologicamente desactivado ou, sem córtex, permitiu o estudo do papel funcional das fibras corticais dirigidas ao núcleo cuneatus. As fibras aferentes primárias activadas pela estimulação de uma zona da pele excitam os neurónios cuneotalâmicos, com os que contactam monossinapticamente e inibem, através de interneurónios intranucleares, outras células cúneotalâmicas vizinhas que recebem informações de outras áreas da pele. Da mesma maneira, as células córtico-cuneatus activadas pela estimulação da pele incrementam a excitação das células cuneotalâmicas com campos receptores sobrepostos e inibem a actividade das restantes. $O$ Córtex exagera desta forma um centro excitado rodeado por uma região inibitória, o que aumenta a capacidade de discriminação táctil, quer espacial, quer temporal durante a exploração e o manuseamento. [REV NEUROL 2001; 33: 448-54]

Palavras chave. Córtex sensitivo-motor. Modulação cortical. Neurónios cúneo-talâmicos. Núcleo cuneatus. Núcleo ventro-postero-lateral talâmico. Transmissão cutânea. 